\title{
RELAXATION OF ${ }^{53} \mathrm{Cr}$ SPIN ECHO SIGNALS IN $\mathrm{Cd}_{0.985} \mathrm{Ag}_{0.015} \mathrm{Cr}_{2} \mathrm{Se}_{4}$
}

\author{
G.N. Abelyashev ${ }^{a}$, V.N. Berzhansky ${ }^{a}$, S.N. PolulyakH ${ }^{a}$ \\ AND N.A. SERGEEV ${ }^{b}$ \\ ${ }^{a}$ Department of Physics, Simferopol University \\ Yaltinskaja 4, 333007 Simferopol, Ukraine \\ ${ }^{b}$ Institute of Physics, University of Szczecin, Wielkopolska 15, 70-451 Szczecin, Poland
}

The frequency dependences of the relaxation times of NMR spin echo signals of quadrupole nuclei ${ }^{53} \mathrm{Cr}$ at $t_{\mathrm{e}}=\tau$ and $t_{\mathrm{e}}=3 \tau$ in ferromagnetic semiconductor $\mathrm{Cd}_{0.985} \mathrm{Ag}_{0.015} \mathrm{Cr}_{2} \mathrm{Se}_{4}$ were investigated at temperature $4.2 \mathrm{~K}$. It was shown that there are two kinds of the quadrupole nuclei ${ }^{53} \mathrm{Cr}$, which have quite different relaxation times. The existence of two kinds of the nuclei ${ }^{53} \mathrm{Cr}$ was connected with doping of the cadmium selenochromite with $\mathrm{Ag}^{+}$ ions.

PACS numbers: 76.20.+q, 76.60.-k, 76.60.Lz

\section{Introduction}

The pulse-NMR method is one of the powerful techniques for the study of the spin dynamics in magnetically ordered materials. In the case of the quadrupole nuclei with spin $I=3 / 2$ (for example ${ }^{53} \mathrm{Cr}$ nuclei), when the quadrupole interactions of the nuclei do not equal zero, the two echo signals may be observed at $t_{\mathrm{e}}=\tau$ and $t_{\mathrm{e}}=3 \tau$ [1-3]. The first echo signal $V_{\tau}$ is the usual Hahn echo and the NMR spectrum $V_{\tau}(\nu)$ recorded with the aid of this echo reflects all NMR spectral lines of the quadrupole nuclei. However, the spectrum NMR $V_{3 \tau}(\nu)$ recorded with the aid of the echo at $t_{\mathrm{e}}=3 \tau$ consists of the NMR resonance frequencies, whose values are determined by the hyperfine magnetic interaction only [1-3]. The aim of this paper is to analyze the relaxation of the $V_{\tau}(\nu)$ and $V_{3 \tau}(\nu)$ echo signals of the quadrupole nuclei ${ }^{53} \mathrm{Cr}$ in ferromagnetic semiconductors $\mathrm{Cd}_{0.985} \mathrm{Ag}_{0.015} \mathrm{Cr}_{2} \mathrm{Se}_{4}$.

*e-mail: roton@ccssu.crimea.ua 


\section{Experimental results and discussion}

The NMR measurements were made on the polycrystalline multidomain sample $\mathrm{Cd}_{0.985} \mathrm{Ag}_{0.015} \mathrm{Cr}_{2} \mathrm{Se}_{4}$ at $T=4.2 \mathrm{~K}$ in zero static external magnetic field. The experimental results are shown in Fig. 1 and Fig. 2. The analysis of the obtained experimental results were provided assuming that the time fluctuations in the electron magnetization $\boldsymbol{M}_{\mathrm{e}}$ due to the fluctuations in the hyperfine magnetic and quadrupole interactions lead to the relaxation of the spin echo signals. Assuming the frequency $\tau_{c e}^{-1}$ of the time fluctuations of $M_{\mathrm{e}}$ is smaller than NMR resonance frequencies $\nu_{i}$ and so remaining in the fluctuating hyperfine magnetic and electric quadrupole Hamiltonians the secular terms only we obtained the following expression for the relaxation rate of the echo signal $V_{\tau}(\nu)$ :

$$
T_{2}^{-1}\left(\tau, \nu_{i}\right)=A+B_{i} \sin ^{2}\left(2 \theta_{0}\right)
$$

where $i=1,2,3 ; \nu_{1}$ is the resonance frequency of the NMR transition $\pm 1 / 2 \longleftrightarrow$ $\mp 1 / 2 ; \nu_{2,3}$ are the resonance frequencies of the NMR transitions $\pm 3 / 2 \longleftrightarrow \pm 1 / 2$. In Eq. (1) the angle $\theta_{0}$ is the angle between the local trigonal axis and a direction of the electron magnetization vector $M_{\mathrm{e}}$. The NMR resonance frequencies $\nu_{i}$ are uniquely determined by the angle $\theta_{0}$. The solid lines shown in Fig. 1 represent the theoretical frequency dependences obtained from the best fit of Eq. (1) to the observed values of $T_{2}(\tau, \nu)$. As is seen, the theoretical curves agree well with the experimental results. In the secular approximation we obtained the following expression for the relaxation of the multiquantum echo signal $V_{3 \tau}\left(\nu_{1}\right)$ :

$$
T_{2}^{-1}\left(3 \tau, \nu_{1}\right)=3 T_{2}^{-1}\left(\tau, \nu_{1}\right) \text {. }
$$

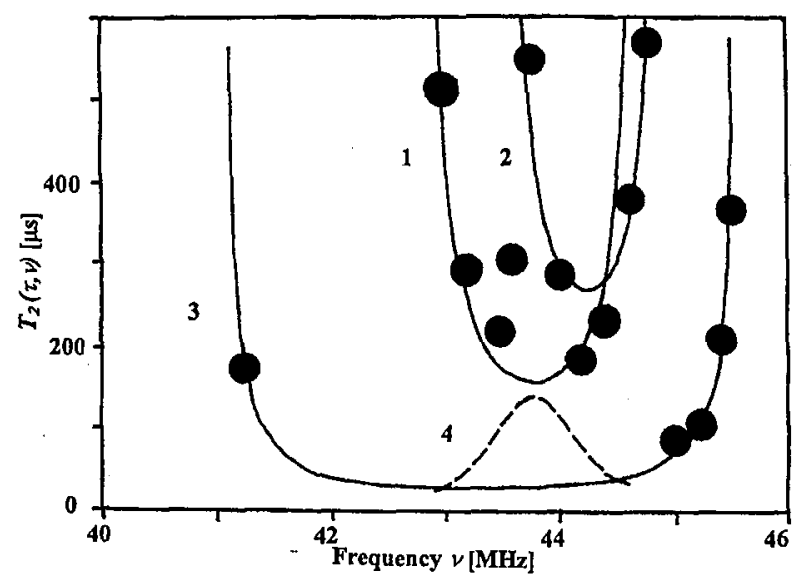

Fig. 1. Frequency dependence of the relaxation time $T_{2}(\tau, \nu)$ of the ${ }^{53} \mathrm{Cr}$ nuclei in $\mathrm{Cd}_{0.985} \mathrm{Ag}_{0.015} \mathrm{Cr}_{2} \mathrm{Se}_{4}$ at $T=4.2 \mathrm{~K}$. The solid lines are the theoretical curves obtained from the best fit of Eq. (1) to the measured values of $T_{2}(\tau, \nu)$. Curve 1 is the dependence $T_{2}\left(\tau, \nu_{1}\right)$; curves 2 and 3 are the dependences $T_{2}\left(\tau, \nu_{2}\right)$ and $T_{2}\left(\tau, \nu_{3}\right)$. Broken line 4 is the theoretical curve $T_{2}\left(\tau, \nu_{1}\right)$ obtained in the nonsecular approximation. 


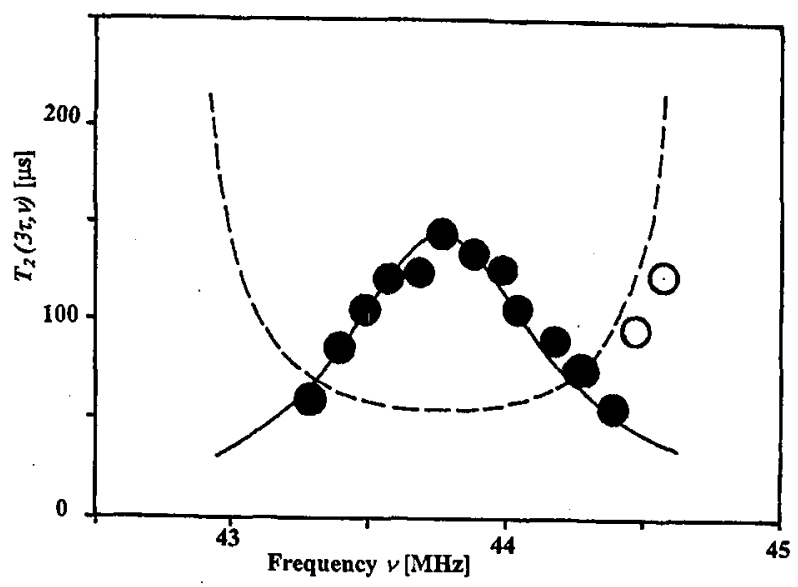

Fig. 2. Frequency dependence of the relaxation time $T_{2}(3 \tau, \nu)$ of the ${ }^{53} \mathrm{Cr}$ nuclei in $\mathrm{Cd}_{0.985} \mathrm{Ag}_{0.015} \mathrm{Cr}_{2} \mathrm{Se}_{4}$ at $T=4.2 \mathrm{~K}$. The solid lines are the theoretical curves obtained from the best fit of Eq. (3) to the measured values of $\mathcal{T}_{2}(3 \tau, \nu)$ (black circles). The broken line is the theoretical curve $\frac{1}{3} T_{2}\left(\tau, \nu_{1}\right)$ obtained with the parameters defined from curve 1 in Fig. 1.

As is seen from Fig. 2 only two experimental points (the open circles) coincide with frequency dependence (2). In order to understand the source of this discrepancy we considered the relaxation of the echo signal at $t_{\mathrm{e}}=3 \tau$ assuming that the time fluctuations of $M_{\mathrm{e}}$ are not small $\left(\tau_{c e}^{-1} \gg \nu_{i}\right)$ and retaining in the fluctuating Hamiltonians the nonsecular terms too. The obtained expression for the relaxation rate of spin echo $V_{3 \tau}\left(\nu_{1}\right)$ has the form

$$
T_{2}^{-1}\left(3 \tau, \nu_{1}\right)=C+D \cos ^{2}\left(2 \theta_{0}\right) \text {. }
$$

As is seen from Fig. 2 the dependence (3) well describes the observed dependence of $T_{2}\left(3 \tau, \nu_{1}\right)$.

The obtained results suggest that in $\mathrm{Cd}_{0.985} \mathrm{Ag}_{0.015} \mathrm{Cr}_{2} \mathrm{Se}_{4}$ there are two kinds of the ${ }^{53} \mathrm{Cr}$ nuclei which have quite different relaxation channels ("secular" and "nonsecular"). The nuclei of the first kind ${ }^{53} \mathrm{Cr}(\mathrm{I})$ give the main contribution to the echo signal $V_{\tau}(\nu)$. In the echo signal $V_{3 \tau}(\nu)$ these nuclei are observed only at $\nu>44.5 \mathrm{MHz}$ (the open circles in Fig. 2). The nuclei of the second kind ${ }^{53} \mathrm{Cr}$ (II) have very short relaxation time $T_{2}(\tau, \nu)$ and so they do not give the contribution to the observed echo signal $V_{\nu}(\tau)$. We may observe these nuclei only with help of the echo $V_{3 \tau}(\nu)$ (the black circles in Fig. 2). In order to understand why the nuclei ${ }^{53} \mathrm{Cr}$ (II) do not give the contribution to the echo signal at $t_{\mathrm{e}}=\tau$ we considered the nonsecular relaxation of the echo signal $V_{\tau}\left(\nu_{1}\right)$. The broken line in Fig. 1 represents the obtained theoretical curve. As is seen the relaxation of the echo signal $V_{\tau}(\nu)$ from the nuclei ${ }^{53} \mathrm{Cr}$ (II) is indeed smaller one for the nuclei ${ }^{53} \mathrm{Cr}$ (I). It is reasonable to assume that the existence of two kinds of the nuclei ${ }^{53} \mathrm{Cr}$ in $\mathrm{Cd}_{0.985} \mathrm{Ag}_{0.015} \mathrm{Cr}_{2} \mathrm{Se}_{4}$ is connected with the doping of $\mathrm{CdCr}_{2} \mathrm{Se}_{4}$ with $\mathrm{Ag}^{+}$ions. The doping of the cadmium selenochromite with silver ions produces, as a result of electric charge compensation, the $\mathrm{Cr}^{4+}$ impurities. The different relaxation channels for the nuclei 
${ }^{53} \mathrm{Cr}(\mathrm{I})$ and ${ }^{53} \mathrm{Cr}$ (II) are probably connected with the dynamical nature of the $\mathrm{Cr}^{4+}$ defects. We assume that electron exchange between the $\mathrm{Cr}^{4+}$ and $\mathrm{Cr}^{3+}$ ions sited inside of the defect region leads to the rapid fluctuations in the local electron magnetization $\left(\tau_{c e}^{-1} \gg \nu_{i}\right)$ so the nuclei ${ }^{53} \mathrm{Cr}$ (II) located in these defect regions "feel" due to the hyperfine and quadrupole interactions the rapidly fluctuating electron magnetization. The rate of local fluctuations of $M_{\mathrm{e}}$ for the nuclei ${ }^{53} \mathrm{Cr}(\mathrm{I})$ which are sited far from defects is small, then $\nu_{i}$ and the relaxation of the echo signals from these nuclei is "secular".

\section{References}

[1] G.N. Abelyashev, V.N. Berzhansky, N.A. Sergeev, Yu.V. Fedotov, Sov. Phys.-JETP 61, 127 (1988).

[2] G.N. Abelyashev, V.N. Berzhansky, N.A. Sergeev, Yu.V. Fedotov, Phys. Lett. A 133, 263 (1988).

[3] G.N. Abelyashev, V.N. Berzhansky, S.N. Polulyakh, N.A. Sergeev, Yu.V. Fedotov, Sov. Phys.-JETP 100, 1981 (1991). 